\title{
Gastrointestinal symptom and stress recall survey in frontline healthcare workers after consumption of a combined quebracho/conker tree/M. balsamea willd extract during the COVID-19 pandemic
}

\author{
Kenneth Brown ${ }^{1}$, Brandi Scott-Hoy ${ }^{1}$ and Bruce P Burnett ${ }^{2 *}$ \\ ${ }^{1}$ KBS Research LLC, 15770 N. Dallas Pkwy, Suite 550, Dallas, TX 75248, USA \\ ${ }^{2}$ Independent Consultant, 128 Virginia Creek Drive, Holly Springs, NC 27540, USA
}

\begin{abstract}
Background. Psychological stress induces gastrointestinal (GI) symptoms such as bloating, constipation, abdominal pain/discomfort and diarrhea sometimes of unknown etiology which impairs quality-of-life $(\mathrm{QoL})$ in affected individuals. After a year of the COVID-19 pandemic, the stress impact on healthcare workers is becoming apparent.

Methods. Thirty-five (35) frontline healthcare workers were surveyed by recall regarding previous GI symptoms, their frequency and whether a combined extract of quebracho, conker tree and M. balsamea Willd (ATRANTIL ${ }^{\circledR}$ ) improved symptomatology, QoL, anxiety and gave them more confidence during the COVID-19 pandemic.

Results. Twenty-eight (28) of 35 participants reported GI distress prior to taking the product with 27 having frequent bloating, abdominal discomfort/pain and constipation as their primary symptoms. After taking the combined extract, $77 \%$ reported improvement in bloating followed by constipation (44\%), abdominal pain/ discomfort (44\%), abdominal distention (38\%) and diarrhea (5\%). Almost $90 \%$ stated improved QoL with reductions in anxiety and increased confidence during the pandemic. There were no adverse events during or after taking the supplement.

Conclusion. The combined extract of quebracho, conker tree and M. balsamea Willd may improve psychological stress-related GI distress, particularly bloating and overall QoL. More attention should be given to frontline healthcare workers under stress-induced somatization with GI involvement and strategies to manage symptomology.
\end{abstract}

\section{Introduction}

Since the coronavirus (SARS-CoV-2) pandemic began, frontline workers in general face the stress of uncertainty and fear in encountering infected individuals in the wider public. Frontline healthcare workers are at particular risk for increasing stress levels when treating and caring for COVID-19 patients. These healthcare professionals experience anxiety, psychological pressure and post-traumatic stress with their associated workloads [1-3]. Post-traumatic stress accompanying the COVID-19 outbreak in healthcare workers also elicits hyperarousal and decreased sleep quality [4]. Post-traumatic stress is known to induce somatization with gastrointestinal (GI) symptoms [5].

For decades, the brain-gut axis has been implicated in disorders of abdominal pain and motility in the GI tract [6]. More recently, researchers and clinicians have shown that there is an interplay between diet, the microbiome and psychological stress on function of the endocrine and nervous systems with regard to digestive distress in functional GI disorders or more recently described as disorders of gut-brain interaction (DGBI) [7]. Irritable bowel syndrome (IBS) and functional dyspepsia are both DGBIs which can be triggered and exacerbated by psychological stress and anxiety $[8,9]$. The multiple factors involved in symptomatology of DGBI have greatly influenced the treatment of these disorders with numerous agents that directly impact the microbiome (i.e., antibiotics, probiotics), stress (i.e., neuromodulatory agents), motility (i.e., peripheral opioid receptor agonists/antagonists) as well as complementary alternative medicine approaches that focus on the brain-gut connection (i.e., dietary modification, dietary supplements, acupuncture, etc) [7,10-12].

Though stress can induce symptoms in patients with diagnosed DGBI, it can also more widely impact GI function in healthy populations confronted with extreme circumstances. Taylor et al. [13] recently described the stress and anxiety associated with COVID-19 in over 6,800 US and Canadian adults as the COVID Stress Syndrome finding that stress and anxiety had increased substantially and contributed to pre-existing psychopathologies. A recent study of over 2,000 United Kingdom citizens found that fatigue and GI symptoms were the most

${ }^{*}$ Correspondence to: Bruce P Burnett, Consultant, KBS Research LLC, 128 Virginia Creek Drive, Holly Springs, NC 27540, USA, Tel: +1-954-299-1105; Email: bburnett33019@yahoo.com

Key words: coronavirus disease 2019 (COVID-19), stress, anxiety, gastrointestinal (GI) symptoms, bloating, constipation, abdominal pain/discomfort

Received: March 15, 2021; Accepted: March 20, 2021; Published: March 27, 2021 
Brown K (2021) Gastrointestinal symptom and stress recall survey in frontline healthcare workers after consumption of a combined quebracho/conker tree/M. balsamea willd extract during the COVID-19 pandemic

predominant somatic effects of the anxiety during the pandemic [14]. Different surveys of healthcare professionals have found that the stress and anxiety can also contribute to GI symptoms. Nurses and nursing students, for example, reported significant increases in symptoms associated with DGBI $[15,16]$. And in a recent survey of Italian healthcare workers $(\mathrm{N}=1,153)$ assessing the effects of the COVID-19 pandemic on psycho-somatic symptoms, Barello et al. [17] found that 37\% experienced GI symptomatology.

A marketed dietary supplement containing a blended extract of quebracho, conker tree and M. balsamea Willd (ATRANTIL ${ }^{\circledR}$ ) has been shown previously to improve bloating, abdominal pain/discomfort and constipation $[18,19]$. With the psychological stress of frontline healthcare workers in mind during the pandemic, ATRANTIL ${ }^{\circledR}$ was provided to a group of US healthcare professionals and the effect of the dietary supplement on GI symptoms, overall quality of life, anxiety and confidence assessed with an online recall survey.

\section{Materials and methods}

Survey design and participants. This was an open-label, non-controlled Healthcare Professional survey conducted using SurveyMonkey ${ }^{\circledR}$ in and around the Dallas-Fort Worth, TX metropolitan area. Thirty-five (35) frontline healthcare professionals participated in the survey. Survey participants were provided with a 30-day supply of an over-the-counter dietary supplement and then asked to answer survey questions. Survey responses represent their recollection prior to and after taking the product. Product consumption and survey participation occurred between June 1, 2020 to August 11, 2020 during the second peak of the coronavirus pandemic in the United States. This survey was carried out in accordance with The Code of Ethics of the World Medical Association (Declaration of Helsinki) for experiments involving humans and was exempt from IRB review. Consent was obtained from all participants for the use of survey data.

Intervention. Participants were asked to take the recommended daily dose of the combined extract containing quebracho, conker Tree and M. balsamea Willd (ATRANTIL ${ }^{\circledR}$, KBS Research LLC, 15660 N. Dallas Parkway, Suite 700, Dallas, TX 75248) for 30 days. There were no restrictions on taking any prescription drugs, over-the-counter products or diet.

\section{Survey questions. Questions used in the survey and descriptor answers were as follows:}

1. Did you have bloating or digestive issues (including abdominal discomfort/pain, diarrhea, constipation) prior to taking ATRANTIL ${ }^{\circledR}$ ? (Yes/No)

2. If so, how often did you have digestive symptoms? (Never/Rarely/ Sometimes/Often/Very Often)

3. If you answered yes to \#1, did your symptoms improve while on ATRANTIL $^{\circledR}$ ? (Yes/No)

4. If you had symptoms improve while on ATRANTIL $^{\circledR}$, what symptoms improved (check all that apply)? (Bloating/Diarrhea/Constipation/ Abdominal Distention/Abdominal Pain and/or Discomfort)

5. Did your Quality of Life improve while on ATRANTIL ${ }^{\circledR}$ (including digestive issues, anxiety, overall health, etc.)? (Not at all/Slightly Improved/Moderately Improved/Greatly Improved/Completed Improved)

6. Were you exposed to COVID-19 during the time you were on ATRANTIL ${ }^{\oplus}$ ? (Yes/No)
7. Knowing that a healthy gut leads to a healthy immune system, did you have more confidence taking care of your patients while taking ATRANTIL? (Not at All/Slightly More Confident/Moderately More Confident/A Great Deal of Confidence/Completely Confident)

Statistical analysis. Data was accumulated and the percentage of patients responding with descriptor answers described above were tallied for each survey question.

\section{Results}

All participants worked in clinical settings as critical, essential frontline workers. Six (6) of 35 participants (17\%) confirmed a previous diagnosis of IBS. Nine (9) of 35 participants (26\%) were in close contact with COVID-19 patients but did not get infected. The majority of survey participants were from gastroenterology practices. The specific roles of each survey participants in the healthcare system are listed in Table 1.

Participation in this survey occurred between June 1, 2020 to August 11, 2020 during the peak of the first wave in positive SARsCoV-2 case infections in Texas and the second peak of the coronavirus pandemic in the United States. A total of 35 participants (26 females, 5 males and 4 unknown) consumed product and answered the survey questions. Participant responses to the survey questions with the descriptor answers are shown in Table 2.

The majority of participants $(80 \%)$ reported GI symptoms prior to taking the blended extract (i.e., bloating, abdominal discomfort and/or pain, diarrhea, constipation) with $77 \%$ percent experiencing these symptoms sometimes to very often. The primary symptoms that improved after taking the dietary supplement in participants were bloating (76\%), constipation (44\%), abdominal distention (38\%) and abdominal pain/discomfort (44\%). Overall, $89 \%$ of participants reported a moderate to complete improvement in quality of life (i.e., less anxiety, overall better health, fewer digestive issues) after taking the product. Knowing that a healthy gut is important for a healthy immune system, $89 \%$ felt that taking the product gave them moderately more to complete confidence. Finally, 91\% (31/34) reported overall improvement in GI symptoms while on the combined extract.

There were no adverse events to the intervention reported over the course of consumption of the product or after while responding to survey questions.

\section{Discussion}

Quebracho extract contains tannins, large delocalized flavonoid structures found in wine [20]. Tannins act to disrupt bacterial lipid bilayers and bind excess hydrogen and methane [21,22], impacting the microbiome by a reduction in gas-producing microbes in the gut and

Table 1. Roles of frontline healthcare worker

\begin{tabular}{|c|c|}
\hline Healthcare Professional Role & Participants (n) \\
\hline Endoscopy Nurse & 13 \\
\hline Endoscopy Technician & 8 \\
\hline Unknown & 4 \\
\hline Acute Care Nurse & 3 \\
\hline Physician & 2 \\
\hline Medical Assistant & 2 \\
\hline Certified Registered Nurse Anesthetist & 1 \\
\hline Endoscopy Check In Office Staff & 1 \\
\hline Oncology Nurse & 1 \\
\hline Total & $\mathbf{3 5}$ \\
\hline
\end{tabular}


Brown K (2021) Gastrointestinal symptom and stress recall survey in frontline healthcare workers after consumption of a combined quebracho/conker tree/M. balsamea willd extract during the COVID-19 pandemic

Table 2. Frontline healthcare worker survey questions with descriptor responses

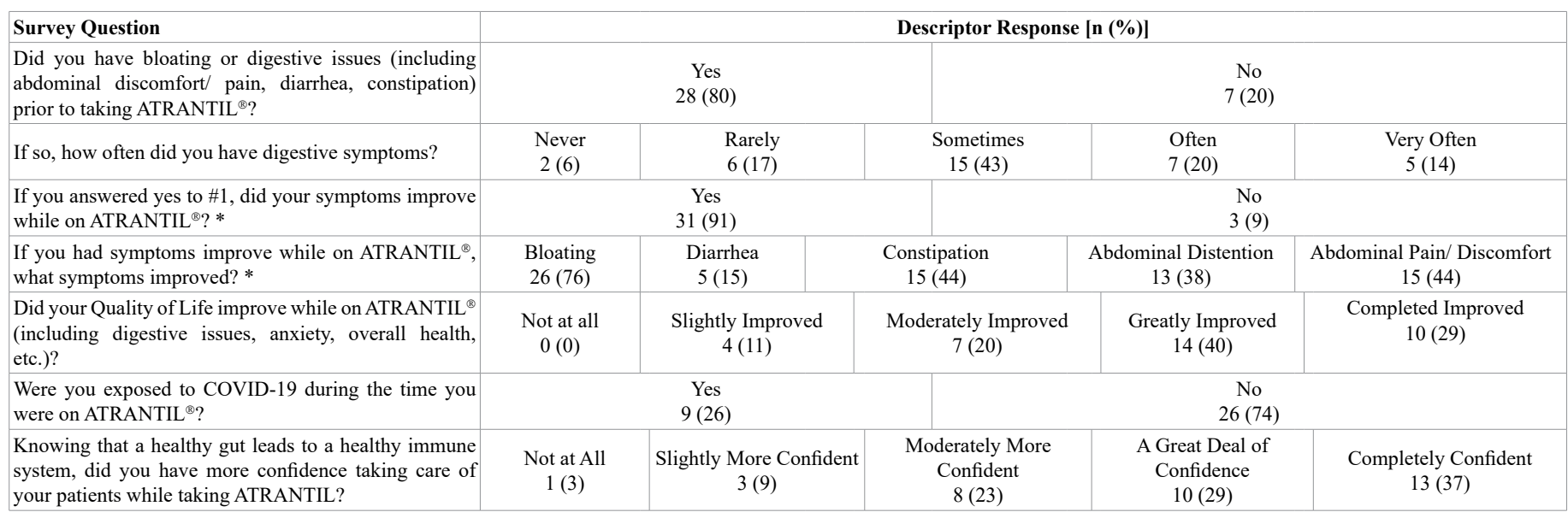

your patients while taking ATRANTIL?

* 34 responded out of 35 participants

the bloating caused by these microorganisms [23]. Conker tree extract contains saponins, also known as escins, which act as antimicrobial agents against methane-producing archaebacteria and also promote intestinal motility [23-25]. Finally, M. balsamea Willd extract contains peppermint oil which has been shown to act as an analgesic and antispasmodic in the gut to reduce abdominal pain/discomfort [26]. This combination extract improves symptoms associated with IBS with constipation (IBS-C); bloating, abdominal pain/discomfort and constipation $[18,19]$.

In addition to the antibacterial activity of polyphenolic compounds of saponins and tannins, the molecules also possess potent antioxidant, anti-inflammatory and antiviral activities $[27,28]$. Escins, or saponins, have a glucocorticoid-like activity and down-regulate nuclear factor $\kappa$-light-chain-enhancer of activated B cells (NFKB) p65 subunit expression, a key transcription factor involved in gene expression of inflammatory cytokines [29]. In a mouse model of indomethacininduced ulceration, escin's antioxidant and anti-inflammatory action alters catalase, glutathione peroxidase and superoxide dismutase activities as well as significantly reduces TNF- $\alpha$, P-selectin, vascular cell adhesion molecule 1 (VCAM-1), malondialdehyde and myeloperoxidase activity, respectively, in gastric tissue [30]. Tannins not only act as a "sink" for hydrogen and methane in the gut, but also as potent antioxidants. Quebracho tannins are not absorbed in an ovine model, but increase antioxidant capacity of purified hepatic tissue and plasma compared to animals not fed tannins [31].

Reactive oxygen species (ROS) are by-products of normal cellular metabolism but when generated in overabundance due to a pathological condition, they contribute to metabolic diseases. In general, the primary ROS-induced inflammatory pathway involves $\mathrm{NF \kappa B}$ activation by $\mathrm{TNF} \alpha$, lipopolysaccharide, thrombin, shear stress within blood vessels, mitochondrial metabolism and dietary factors such as elevated fatty acid and glucose intake [32]. Though endogenous antioxidants in the body respond to ROS challenge, dietary antioxidants, including vitamins $\mathrm{C}, \mathrm{D}$ and $\mathrm{E}$ as well as carotenoids and polyphenols supplement the body's antioxidant capabilities both to reduce the risk for and mitigate damage in cardiovascular disease, chronic obstructive pulmonary disease, inflammatory bowel disease and certain cancers [33]. The highly polymerized quebracho tannins are not highly digestible in the human gut, but bind dietary glucose from starch which helps mitigate elevated blood glucose activation of $\mathrm{NF \kappa B}$-induced inflammation [34]. Colonic microbial digestion of the polymerized tannins leads to a more anti-inflammatory gut microbiome compared to diets with limited polyphenol intake [35]. Finally, a recent in vitro model of digestion demonstrates that quebracho tannins [36] yield not only short chain fatty acids (i.e., propanoic and butyric acids) which can support growth of beneficial microbiota, but also the release of anti-inflammatory flavonoids such as epicatechin gallate and gallocatechin that are more readily absorbed into systemic circulation [37]. The antioxidant properties of these and related polyphenols which down-regulate inflammatory pathways in the body may provide some measure of protection against certain diseases and the impact of viral infection.

It has also long been known that polyphenolic compounds improve antioxidant and anti-inflammatory status linked to lower rates of cardiometabolic disorders $[28,38]$ and better outcomes in certain cancers $[28,39,40]$. In addition, polyphenols also mitigate the effects of certain viral infections [41-43]. Multiple natural compounds and extracts including polyphenols inhibit in vitro growth and plaque formation for SARS-CoV and MERS as well as seasonal coronaviruses [44].

The hypoxic phase of the SARS-CoV-2 infection and COVID-19 disease with pneumonia contributes to a ROS-mediated cytokine upregulation, increased inflammation in endothelial tissue and subsequent coagulation in blood vessels [45-47]. Many different polyphenolic compounds reduce ROS-induced inflammation in respiratory viral infection models of influenza and respiratory syncytial virus [48]. The potential protective effects of polyphenols against respiratory virusinduced ROS-mediated inflammatory disease is intriguing, though studies need to be conducted examining nutritional and oxidative status as a marker for more severe COVID-19 outcomes.

\section{Conclusion}

In IBS and inflammatory bowel disease, psychological stress induces permeability of the intestinal epithelium, a contributor to systemic inflammation [49-51]. A small study $(\mathrm{N}=73)$ of healthy older adults for self-reported GI symptoms (i.e., diarrhea, constipation, indigestion, abdominal pain, reflux) also demonstrates a link between psychological stress and a release of higher zonulin levels in the plasma, a marker for intestinal permeability [52]. Polyphenols in combination with other natural molecules such as analgesic peppermint provide a way to reduce psychological stress-related GI dysfunction by increasing 
Brown K (2021) Gastrointestinal symptom and stress recall survey in frontline healthcare workers after consumption of a combined quebracho/conker tree/M. balsamea willd extract during the COVID-19 pandemic

the body's antioxidant capacity and relieving GI symptoms while maintaining a healthy gut.

The current survey results in a frontline healthcare worker population suggest that the combined extract of quebracho, conker tree and M. balsamea Willd reduces both stress levels in response to the coronavirus pandemic as well as stress-induced GI symptoms to improve their overall quality of life and confidence. Results herein are viewed in the context that the survey is conducted by recall and inaccuracies may exist. A larger, controlled study analyzing specific markers of gut permeability and symptomology is warranted to delineate the exact mechanism by which this dietary supplement improves GI symptoms during elevated periods of psychological stress.

\section{References}

1. Park C, Hwang JM, Jo S, Seong Jin B, Joon S, et al. (2020) COVID-19 outbreak and its association with healthcare workers' emotional stress: A cross-sectional study. $J$ Korean Med Sci 35: e372. [Crossref]

2. Preti E, Di Mattei V, Perego G, Ferrari F, Mazzetti M, et al. (2020) The psychological impact of epidemic and pandemic outbreaks on healthcare workers: rapid review of the evidence. Curr Psychiatry Rep 22: 43. [Crossref]

3. Zhu Z, Xu S, Wang H, Liu Z, Wu J, et al. (2020) COVID-19 in Wuhan: Sociodemographic characteristics and hospital support measures associated with the immediate psychological impact on healthcare workers. EClinical Medicine 24: 100443.

4. Yin Q, Sun Z, Liu T, Ni X, Deng X, et al. (2020) Posttraumatic stress symptoms of health care workers during the corona virus disease 2019. Clin Psychol Psychother 27: 384-395. [Crossref]

5. Gradus JL, Farkas DK, Svensson E, Ehrenstein V, Lash TL, et al. (2017) Posttraumatic stress disorder and gastrointestinal disorders in the danish population. Epidemiology 28: 354-360. [Crossref]

6. Camilleri M (1990) Disorders of gastrointestinal motility in neurologic diseases. Mayo Clin Proc 65: 825-846. [Crossref]

7. Törnblom H, Drossman DA (2018) Psychotropics, antidepressants, and visceral analgesics in functional gastrointestinal disorders. Curr Gastroenterol Rep 20: 58. [Crossref]

8. Talley NJ, Holtmann G, Walker MM. Therapeutic strategies for functional dyspepsia and irritable bowel syndrome based on pathophysiology. J Gastroenterol 50: 601-613. [Crossref]

9. Talley NJ, Ford AC (2015) Functional dyspepsia. N Engl J Med 373: 1853-1863.

10. Dossett ML, Cohen EM, Cohen J (2017) Integrative medicine for gastrointestinal disease. Prim Care 44: 265-280. [Crossref]

11. Yoon SL, Grundmann O, Smith KF, Mason SR. Dietary supplement and complementary and alternative medicine use are highly prevalent in patients with gastrointestinal disorders: Results from an online survey. J Diet Suppl 16: 635-648. [Crossref]

12. Brenner DM, Sayuk GS (2020) Current US food and drug administration-approved pharmacologic therapies for the treatment of irritable bowel syndrome with diarrhea. Adv Ther 37: 83-96. [Crossref]

13. Taylor S, Landry CA, Paluszek MM, Thomas AF, Dean M, et al. COVID stress syndrome: Concept, structure, and correlates. Depress Anxiety 37: 706-714. [Crossref]

14. Shevlin M, Nolan E, Owczarek M, McBride O, Jamie M, et al. (2020) COVID-19related anxiety predicts somatic symptoms in the UK population. Br J Health Psychol 25: 875-882. [Crossref]

15. Lee EY, Mun MS, Lee SH, Cho HS (2011) Perceived stress and gastrointestinal symptoms in nursing students in Korea: A cross-sectional survey. BMC Nurs 10: 22. [Crossref]

16. Koh SJ, Kim M, Oh DY, Byeong Gwan K, Kook Lae L, et al. (2014) Psychosocial stress in nurses with shift work schedule is associated with functional gastrointestinal disorders. J Neurogastroenterol Motil 20: 516-522. [Crossref]

17. Barello S, Palamenghi L, Graffigna G (2020) Burnout and somatic symptoms among frontline healthcare professionals at the peak of the Italian COVID-19 pandemic Psychiatry Res 290: 113129. [Crossref]
18. Brown K, Scott-Hoy B, Jennings LW (2015) Efficacy of a quebracho, conker tree, and M. balsamea Willd blended extract in a randomized study in patients with irritable bowel syndrome with constipation. $J$ Gastroenterol Hepatol Res 4: 9.

19. Brown K, Scott-Hoy B, Jennings LW (2016) Response of irritable bowel syndrome with constipation patients administered a combined quebracho/conker tree/M balsamea Willd extract. World J Gastrointest Pharmacol Ther 7: 463-468. [Crossref]

20. Bertoldi D, Santato A, Paolini M, Barbero A, Camin F, et al. (2014) Botanica traceability of commercial tannins using the mineral profile and stable isotopes. J Mass Spectrom 49: 792-801. [Crossref]

21. Nakayama T, Hashimoto T, Kajiya K, Kumazawa S (2000) Affinity of polyphenols for lipid bilayers. Biofactors 13:147-151. [Crossref]

22. Hook SE, Wright AD, McBride BW (2010) Methanogens: Methane producers of the rumen and mitigation strategies. Archaea 2010: 945785. [Crossref]

23. Díaz Carrasco JM, Cabral C, Redondo LM, et al. (2017) Impact of chestnut and quebracho tannins on rumen microbiota of bovines. Biomed Res Int 2017: 9610810.

24. Fu F, Hou Y, Jiang W, Ronghua W, Liu K, et al. (2005) Escin: Inhibiting inflammation and promoting gastrointestinal transit to attenuate formation of postoperative adhesions. World J Surg 29: 1614-1620. [Crossref]

25. Guo YQ, Liu JX, Lu Y, Denman SE, McSweeney CS, et al. (2008) Effect of tea saponin on methanogenesis, microbial community structure and expression of mcrA gene, in cultures of rumen micro-organisms. Lett Appl Microbiol 47: 421-426. [Crossref]

26. Ford AC, Moayyedi P, Lacy BE, Lembo AJ, Saito YA, et al. (2014) Task force on the management of functional bowel disorders. American college of gastroenterology monograph on the management of irritable bowel syndrome and chronic idiopathic constipation. Am J Gastroenterol 109 Suppl 1: S2-26; quiz S27. [Crossref]

27. Naithani R, Huma LC, Holland LE, Shukla D, McCormick DL, et al. (2008) Antiviral activity of phytochemicals: a comprehensive review. Mini Rev Med Chem 8: 11061133. [Crossref]

28. Yahfoufi N, Alsadi N, Jambi M, Matar C (2018) The immunomodulatory and antiinflammatory role of polyphenols. Nutrients 10: 1618. [Crossref]

29. Wang H, Zhang L, Jiang N, Zhenhua W, Yating C, et al. (2013) Anti-inflammatory effects of escin are correlated with the glucocorticoid receptor/NF-KB signaling pathway, but not the COX/PGF2 $\alpha$ signaling pathway. Exp Ther Med 6: 419-422. [Crossref]

30. Wang T, Zhao S, Wang Y, Yang Y, Yao L, et al. (2014) Protective effects of escin against indomethacin-induced gastric ulcer in mice. Toxicol Mech Methods 24: 560566. [Crossref]

31. López-Andrés P, Luciano G, Vasta V, Trevor MG, Luisa B, et al. (2013) Dietary quebracho tannins are not absorbed, but increase the antioxidant capacity of liver and plasma in sheep. Br J Nutr 110: 632-639. [Crossref]

32. Forrester SJ, Kikuchi DS, Hernandes MS, Qian X, Kathy KG (2018) Reactive oxygen species in metabolic and inflammatory signaling. Circ Res 122: 877-902.

33. Liu Z, Ren Z, Zhang J, Chia-Chen C, Eswar K, et al. (2018) Role of ROS and nutritional antioxidants in human diseases. Front Physiol 9: 477. [Crossref]

34. Amoako D, Awika JM (2016) Polyphenol interaction with food carbohydrates and consequences on availability of dietary glucose. Curr Opinion Food Sci 8: 14-18.

35. Thumann TA, Pferschy-Wenzig EM, Moissl-Eichinger C, Bauer R (2019) The role of gut microbiota for the activity of medicinal plants traditionally used in the European Union for gastrointestinal disorders. J Ethnopharmacol 245: 112153. [Crossref]

36. Molino S, Fernández-Miyakawa M, Giovando S, Rufián-Henares JA (2018) Study of antioxidant capacity and metabolization of quebracho and chestnut tannins through in vitro gastrointestinal digestion-fermentation. J Functional Foods 49: 188-195.

37. Kürbitz C, Heise D, Redmer T, Freya G, Alexander A, et al. (2011) Epicatechin gallate and catechin gallate are superior to epigallocatechin gallate in growth suppression and anti-inflammatory activities in pancreatic tumor cells. Cancer Sci 102: 728-734. [Crossref]

38. Soory M (2012) Nutritional antioxidants and their applications in cardiometabolic diseases. Infect Disord Drug Targets 12: 388-401. [Crossref]

39. Darvesh AS, Bishayee A (2013) Chemopreventive and therapeutic potential of tea polyphenols in hepatocellular cancer. Nutr Cancer 65: 329-344. [Crossref]

40. Losada-Echeberría M, Herranz-López M, Micol V, Barrajón-Catalán E (2017) Polyphenols as promising drugs against main breast cancer signatures. Antioxidants (Basel) 6: 88. [Crossref] 
Brown K (2021) Gastrointestinal symptom and stress recall survey in frontline healthcare workers after consumption of a combined quebracho/conker tree/M. balsamea willd extract during the COVID-19 pandemic

41. Andres A, Donovan SM, Kuhlenschmidt MS (2009) Soy isoflavones and virus infections. J Nutr Biochem 20: 563-569. [Crossref]

42. Bahramsoltani R, Sodagari HR, Farzaei MH, Amir Hossein A, Maziar G, et al. (2016) The preventive and therapeutic potential of natural polyphenols on influenza. Expert Rev Anti Infect Ther 14: 57-80. [Crossref]

43. Furushima D, Ide K, Yamada H (2018) Effect of tea catechins on influenza infection and the common cold with a focus on epidemiological/clinical studies. Molecules 23: 1795. [Crossref]

44. Mani JS, Johnson JB, Steel JC, Daniel AB, Paul MN, et al. (2020) Natural productderived phytochemicals as potential agents against coronaviruses: A review. Virus Res 284: 197989. [Crossref]

45. Cecchini R, Cecchini AL (2020) SARS-CoV-2 infection pathogenesis is related to oxidative stress as a response to aggression. Med Hypotheses 143: 110102. [Crossref]

46. Morris G, Bortolasci CC, Puri BK, Lisa O, Wolfgang M, et al. (2020) The pathophysiology of SARS-CoV-2: A suggested model and therapeutic approach. Life Sci 258: 118166. [Crossref]
47. Nasi A, McArdle S, Gaudernack G, Westman G, Melief C, et al. (2020) Reactive oxygen species as an initiator of toxic innate immune responses in retort to SARS-CoV-2 in an ageing population, consider $\mathrm{N}$-acetylcysteine as early therapeutic intervention. Toxicol Rep 7: 768-771. [Crossref]

48. Khalil A, Tazeddinova D (2020) The upshot of polyphenolic compounds on immunity amid COVID-19 pandemic and other emerging communicable diseases: An appraisal. Nat Prod Bioprospect 10: 411-429. [Crossref]

49. Deiteren A, de Wit A, van der Linden L, De Man JG, Pelckmans PA, et al. (2016) Irritable bowel syndrome and visceral hypersensitivity: risk factors and pathophysiological mechanisms. Acta Gastroenterol Belg 79: 29-38. [Crossref]

50. Bernstein CN (2017) The brain-gut axis and stress in inflammatory bowel disease. Gastroenterol Clin North Am 46: 839-846. [Crossref]

51. Oligschlaeger Y, Yadati T, Houben T, Claudia Maria Condello O, Ronit Shiri S, et al. (2019) Inflammatory bowel disease: A stressed "Gut/Feeling". Cells 8: 659. [Crossref]

52. Ganda Mall JP, Östlund-Lagerström L, Lindqvist CM, Algilani S, Rasoal D, et al (2018) Are self-reported gastrointestinal symptoms among older adults associated with increased intestinal permeability and psychological distress? BMC Geriatr 18: 75 .

Copyright: (C2021 Brown K. This is an open-access article distributed under the terms of the Creative Commons Attribution License, which permits unrestricted use, distribution, and reproduction in any medium, provided the original author and source are credited. 